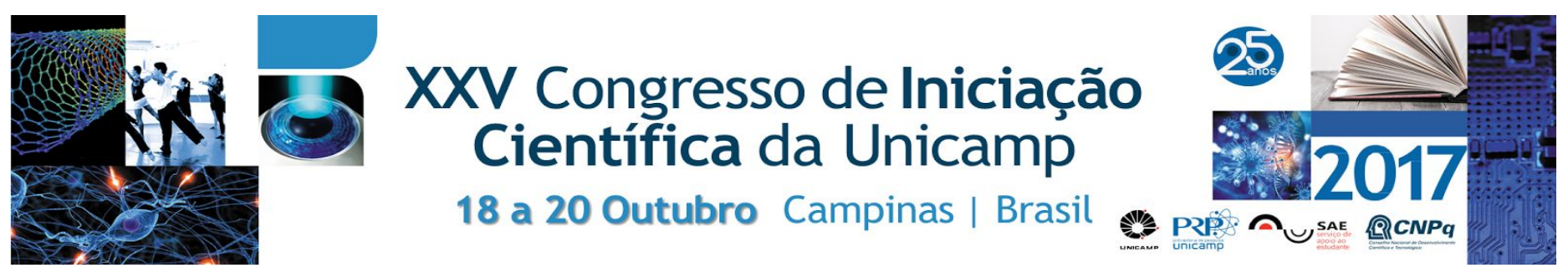

\title{
Sistema combinado anaeróbio-aeróbio tratando esgoto sanitário contendo fenol: avaliação de desempenho
}

\section{Cláudia N. Paltronieri, Edson A. A. Nour, Raphael Fuzza Duarte* \\ Abstract}

The purpose of this study was to physically and chemically evaluate the final residue generated by a combined reactor treating sanitary sewage containing high phenol loading. In addition, it is aimed at the degradation of phenol using the anaerobic-aerobic system consisting of an anaerobic filter (AF), followed by submerged aerated biofilter (BAS) and secondary decanter (DS), operating in order to reach the limit for inhibition of the biomass and subsequent adoption of the internal recirculation to achieve the reversal of the biomass inhibition.

\section{Palavras-chave:}

Sistema combinado,fenol, variáveis físicas e químicas.

\section{Introdução}

A combinação dos processos anaeróbios e aeróbios trazem grandes vantagens para o sistema. $\mathrm{O}$ anaeróbio possui baixa produção de sólidos, tolerância a cargas orgânicas e baixo gasto energético, porém seu produto ainda possui quantidades consideráveis de material carbonáceo e nitrogenado, fazendo com que se encaixe o sistema aeróbio que, por mais que tenha um gasto energético maior, degrada de modo mais eficiente a matéria orgânica e os compostos nitrogenados.

Além disso, é importante salientar na pesquisa o estudo em relação ao fenol, bastante presente em fontes pontuais como em efluentes industriais e que podem ser prejudiciais para águas de abastecimento, seres vivos aquáticos e para os seres humanos, causando náuseas, diarréia e até a morte.

Assim, o trabalho tem como objetivo analisar química e fisicamente o esgoto sanitário contendo fenol após a passagem deste pelo sistema combinado contendo filtro anaeróbio, biofiltro aerado submerso e decantador secundário, fazendo uma análise do produto resultante e do reator utilizado.

\section{Resultados e Discussão}

As análises foram divididas em 3 etapas e colocados os valores médios e desvios padrões em cada uma, de acordo com as tabelas, medidos em 4 pontos diferentes do sistema: EB(efluente bruto), FA (após o filtro anaeróbio), BAS (após o biofiltro aerado submerso) e DS (após o decantador secundário).

A segunda e terceira etapa foi acrescida de fenol em uma quantidade gradativa.

Tabela 1: Análise de desempenho da primeira etapa

\begin{tabular}{|c|c|c|c|c|c|c|c|c|}
\hline \multirow[t]{2}{*}{ Variável } & \multicolumn{2}{|c|}{ EB } & \multicolumn{2}{|c|}{ FA } & \multicolumn{2}{|c|}{ BAS } & \multicolumn{2}{|c|}{ DS } \\
\hline & MED & DP & MED & DP & MED & $D P$ & MED & DP \\
\hline Temperatura $\left({ }^{\circ} \mathrm{C}\right)$ & 26 & 2 & 26 & 2 & 25 & 1 & 25 & 1 \\
\hline AOV (mg HAC..-1) & 126,5 & 54,7 & 143,4 & 59,9 & 47,1 & 56,6 & 39,2 & 36,0 \\
\hline $\mathrm{AP}\left(\mathrm{mg} \mathrm{CaCO}_{3} \mathrm{~L}^{-1}\right)$ & 141,7 & 27 & 152,2 & 28,1 & 54,7 & 65,9 & 53,1 & 70,7 \\
\hline $\mathrm{AT}\left(\mathrm{mg} \mathrm{CaCO}_{3} \mathrm{~L}^{-1}\right)$ & 230,3 & 23,7 & 230,6 & 23,2 & 70,5 & 92,3 & 73,6 & 92,3 \\
\hline Al/AP & - & - & 0,6 & 0,2 & - & - & - & - \\
\hline $\mathrm{DQO}\left(\mathrm{mg} \mathrm{O}_{2} \mathrm{~L}^{-\mathrm{L}^{-1}}\right)$ & 607,5 & 153,6 & 427,7 & 119,0 & 187,2 & 147,5 & 146,2 & 118,6 \\
\hline $\mathrm{OD}\left(\mathrm{mg} \mathrm{L}^{\left.\mathrm{L}^{-1}\right)}\right.$ & 0,8 & 0,4 & 2,3 & 1,6 & 5,9 & 2,0 & 5,6 & 2,3 \\
\hline $\mathrm{C}_{\mathrm{O}} \mathrm{H}_{5} \mathrm{OH}\left(\mathrm{mg} \mathrm{L}^{-1}\right)$ & - & - & - & & - & - & - & - \\
\hline $\mathrm{pH}$ & 7,4 & 0,3 & 7,7 & 0,3 & 7,0 & 0,8 & 7,2 & 0,6 \\
\hline $\begin{array}{l}\text { Condutividade } \\
\text { eletrica }\left(\mu \mathrm{S} . \mathrm{cm}^{-1}\right)\end{array}$ & 982,8 & 101,3 & 999,5 & 123,4 & 790,7 & 82,0 & 799,3 & 95,9 \\
\hline $\mathrm{SST}\left(\mathrm{mg} \cdot \mathrm{L}^{-1}\right)$ & 159,3 & 75,7 & 74,1 & 29,5 & 46,9 & 45,1 & 33,8 & 50,2 \\
\hline $\mathrm{SSV}\left(\mathrm{mg} \mathrm{L}^{-1}\right)$ & 134,0 & 70,7 & 61,8 & 27,6 & 44,6 & 42,7 & 32,1 & 45,7 \\
\hline $\mathrm{SSF}\left(\mathrm{mg} \mathrm{LL}^{-1}\right)$ & 25,4 & 18,9 & 12,3 & 10,9 & 2,2 & 3,2 & 1,7 & 5,2 \\
\hline
\end{tabular}

Tabela 2: Análise de desempenho da segunda etapa

\begin{tabular}{|c|c|c|c|c|c|c|c|c|}
\hline \multirow[t]{2}{*}{ riável } & \multicolumn{2}{|c|}{ EB } & \multicolumn{2}{|c|}{ FA } & \multicolumn{2}{|c|}{ BAS } & \multicolumn{2}{|c|}{ DS } \\
\hline & MED & DP & MED & DP & MED & DP & MED & DP \\
\hline nperatura $\left({ }^{\circ} \mathrm{C}\right)$ & & & 24 & & & 1 & 25 & 1 \\
\hline AOV (mg HACL-1-1) & 125.1 & 60,1 & 88.1 & $\begin{array}{l}43,4 \\
\end{array}$ & 3.3 & 55,7 & & 11.1 \\
\hline $\mathrm{AP}\left(\mathrm{mg} \mathrm{CaCO}_{2} \mathrm{~L}^{-1}\right)$ & 98,2 & & 121,7 & 37,8 & 47,2 & 51,4 & 64,7 & 59,2 \\
\hline $\mathrm{AT}\left(\mathrm{mg} \mathrm{CaCO}_{3} \mathrm{~L}\right.$ & 177,3 & 33 & 195,3 & 35,2 & 2,5 & 62,5 & 83,5 & 70,9 \\
\hline & & & & & & & & \\
\hline $\mathrm{DQO}\left(\mathrm{mg} \mathrm{O}_{2} \mathrm{~L}^{-1}\right)$ & 2199,2 & 1849,3 & 953,8 & 485,4 & 391,3 & 329,2 & 163,4 & 139,9 \\
\hline $\mathrm{OD}(\mathrm{mg}$ & 1,4 & 1,1 & 1,0 & 0.4 & 4.7 & 2,1 & 4,8 & 2,1 \\
\hline $\mathrm{C}_{6} \mathrm{H}_{5} \mathrm{OH}\left(\mathrm{mg} \mathrm{L}^{-4}\right)$ & 303,3 & 204,4 & 252,3 & 163,3 & 15, & & & \\
\hline $\mathrm{pH}$ & 7,3 & 0,3 & 7,5 & 0,3 & 6,8 & 0,6 & 7,2 & 0,5 \\
\hline $\begin{array}{l}\text { Condutiv } \\
\text { eletrica }\end{array}$ & 704,8 & 97,5 & 769,8 & 107,4 & 556,4 & 116,3 & 570,0 & 120,0 \\
\hline SST In & 554 & & 112,3 & 170,2 & 121,1 & 110,2 & 83,1 & 179,0 \\
\hline $\operatorname{ssv}(r)$ & 493 & 339, & 102,8 & 159,8 & 104,9 & 108 & 76,2 & 162,1 \\
\hline $\operatorname{SSF}\left(m g \mathrm{~L}^{-1}\right)$ & 61,0 & 61,7 & 9,5 & 15,5 & 16,3 & 33,6 & 7 & 17,4 \\
\hline
\end{tabular}

Tabela 3: Análise de desempenho da terceira etapa

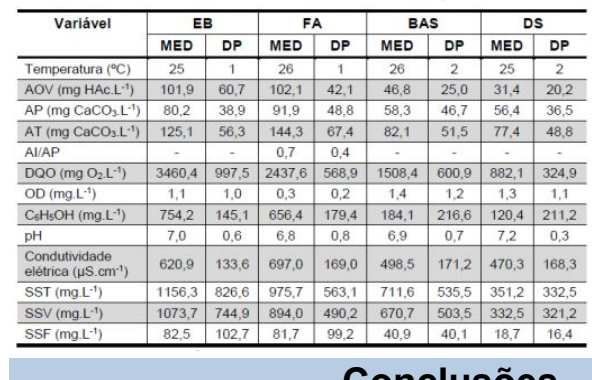

\section{Conclusões}

Observou-se através dos valores médios que o BAS apresentou melhor remoção de $\mathrm{DQO}$ na etapa $A$ e de fenol tanto na etapa $B$ quanto na $C$, mas ao mesmo tempo observou-se a ineficiência em remover SST na etapa B.

No sistema, a remoção da DQO,SST e fenol é mais eficiente na etapa B.

\section{Agradecimentos}

Agradeço ao PIBIC pelo auxílio fomento, ao meu orientador Edson Nour pelos grandes esclarecimentos a respeito da minha área de tanto interesse profissional. Agradeço também à minha namorada Débora Rossetto e à mestranda Cláudia Paltronieri que tanto me auxiliaram na busca pelo resultado.

ADÁRIO, M.L. Degradação de formaldeído em sistema combinado anaeróbio-aeróbio com recirculação interna. Dissertação (Mestrado) Universidade Estadual de Campinas, Faculdade de Engenharia Civil, Arquitetura e Urbanismo. Campinas, SP, 2016. 107p

FOCO, M.L.R. Filtro anaeróbio seguido de biofiltro aerado submerso: produção de biomassa, conversão de matéria orgânica e de nitrogênio. Dissertação de Mestrado. Universidade Estadual de Campinas, 109 p Campinas, 2010. 